\title{
Biodegradable, Non-Enzymatic, Non-Invasive and Cost-Effective Ethanol Sensing Material and Its Electrochemical Characteristics
}

\author{
Preethi Ramadoss $^{1} \cdot$ Agilan Perumal $^{2} \cdot$ Rajendran Nallaiyan $^{2} \cdot$ Arivuoli Dakshanamoorthy $^{1}$ \\ ${ }^{1}$ Crystal Growth Centre, Anna University, Chennai, India. \\ ${ }^{2}$ Department of Chemistry, Anna University, Chennai, India.
}

ABSTRACT

A facile synthesis of cellulose composite biodegradable material for ethanol sensing has been presented. The lowest detection limit of ethanol was found to be $0.34 \mathrm{mM}$. Electrochemical analyses were performed with artificial sweat as the electrolyte; the material is eco-friendly and degrades in soil within 15 days and it is stable in artificial sweat for up to 12 days. It is a non-enzymatic ethanol sensing material made of a polyelectrolytic cellulose derivative composite. This work highlights the electrochemical properties of the synthesized material.

(C) 2021 JMSSE $\cdot$ INScienceIN. All rights reserved
ARTICLE HISTORY

Received 01-03-2021

Revised 01-04-2021

Accepted 02-04-2021

Published 22-04-2021

\section{KEYWORDS}

Cellulose composite

Biodegradable

Non-invasive

Non-enzymatic

Ethanol sensor

\section{Introduction}

Alcohol consumption has been proven to cause liver cirrhosis, epilepsy, road accidents and other violent crimes. This demands an immediate requirement for methods to monitor alcohol abuse. About $0.7 \%$ of the ethanol consumed is observed to get excreted through the breath, which is then detected by a breath analyzer. However, it is also found that an additional $0.1 \%$ of the ethanol consumed is transported by sweat to the surface of the skin. Ethyl glucuronide (EtG) and ethyl sulphate (EtS) have been investigated recently as markers for ethanol detection. Flexible substrates made of polyimide, polyethylene naphthalate, polyethylene terephthalate, polytetrafluoroethylene (Teflon), etc., have been extensively employed in making printed circuit boards (PCBs). In the recent past the usage of these materials have been exploited in the field of electrochemical devices for the fabrication of flexible and wearable sensors[1-4]. EtG is a minor metabolite of alcohol that is an outcome of the reaction between alcohol and glucuronic acid found in the liver, which is a substance that detoxifies the drugs. Panneer Selvam et.al [5] demonstrated a wearable sensor prototype for detecting EtG from human sweat using labelfree electrochemical chemi-impedance sensing methodology by incorporating a single capture immunoassay sensing method onto the surface of polyimide that facilitates chemiresistive signal transduction. Gold and zinc oxide electrodes were integrated on the surface of the flexible polyimide to detect EtG from human sweat in a wearable system [5]. Penza et al. [6] have demonstrated the integration of single-walled carbon nanotubes (SWCNTs) onto a quartz crystal microbalance (QCM) and a standard silica optical fiber (SOF) sensor for detecting alcohol at room temperature. In this study, an optical microfiber sensor is coated with a conducting polymer such as polyaniline (PAni) that acts as the detector. PAni was coated on the surface of the microfiber by drop coating method. The sensor prototype exhibits wavelength shifts in the output spectrum with response to different types of alcohols at various concentrations. The output response was correlated with the solution's refractive index and the number of alkyl groups of alcohol present [7]. Vijayan et al., [8] developed a Smart Helmet, which ensures that the person wearing the helmet has non- alcoholic breath while driving. The team has demonstrated this by affixing a transmitter on the helmet and a receiver on the bike, and there is a switch to ensure that the person is wearing of helmet. An alcohol sensor is placed near the mouth of the driver on the inside of the helmet in order to detect the presence of alcohol from breath. The data to be transferred was coded with radiofrequency (RF) encoder and it is then transmitted through radio frequency transmitter which is then detected by a receiver fixed on the bike that prevents the engine from starting if the conditions were violated. Acetaldehyde is the first metabolite of the ethanol which is carcinogenic that is responsible for cirrhosis on the liver and the upper aero-digestive tract [9]. Besides, it was also observed that a significant increase in risk of cancers of the upper aerodigestive tract was associated with light alcohol drinking in Asian countries, where $28-45 \%$ of the population have found to possess a mutation in the gene ALDH2 [10, 11]. Many other factors in addition to acetaldehyde are also responsible for causing cancer in individual who are alcoholic [12] such as increase of estrogen and androgen levels in women that could lead to the onset of breast cancer [13], or the alcohol-related immunodeficiency and immunosuppression that could facilitate carcinogenesis in various other organs. In addition to these, ethanol-related folate mal-absorption and deficiency can cause different forms of cancer, of which colon cancer is the most commonly described [14]. Similarly, alcohol may cause lesions on the epithelium of the upper digestive and respiratory tract, and favour the absorption of various carcinogenic substances [15].

Alcoholic beverages in general are known to increase the risk of colorectal cancer. Fedirko et al., found that there is a significant heterogeneity between men and women [16], 
according to their research they found there was not much of a difference in alcohol-related cancer between men and women. Cai et al., [15] have reported that they have found evidence that alcohol is associated with an increased risk of melanoma. The actual mechanisms for the harmful effect of alcohol drinking linked to skin cancer are not clear. However, in the presence of UV radiation, it is observed that the alcohol intake can considerably intensify the cellular damage and subsequently lead to skin cancers [17]. The present work demonstrates the use of a flexible and biodegradable cellulose composite material exhibiting properties that could detect the ethanol concentration in the artificial sweat. The lowest detection limit of $0.34 \mathrm{mM}$ of ethanol was found with chrono-amperometric analysis. Although the detection range was not so comparable to other work done by Ajan Panneerselvam et al. whose detection limit for Au electrode showed $1 \mu \mathrm{g} / \mathrm{L}$ and for $\mathrm{ZnO}$ which showed a detection limit of as low as $0.001 \mu \mathrm{g} / \mathrm{L}$ [5] and in the work done by Hauke et al., [18] which showed a detection limit of $0.014 \mathrm{mM}$, in this work we focus more on non-invasive, non-enzymatic and a completely biodegradable sensor which showed that it is good enough for a proof-of-concept model. Earlier Nur Cicek Kekec er.al, (2013) [19] have reported about a conducting polymer and its ethanol sensing properties. They have covalently immobilized alcohol oxidase on the surface of a graphite electrode which is polymerized with 9-methyl-9Hcarbozole-3-carbohydrazine (MCCH).Alternatively, Debasis Maity et.al., (2018) [20] reported about a layer-by-layer spray coating of multi-walled carbon nanotubes (MWCNT) and PVA on commercially available cotton fabrics and subsequently the ethanol gas sensing properties were determined at room temperature.

This work is in continuance with our previous work on the same material for its glucose sensing ability studied and presented in detail[21].

\section{Experimental}

\section{Materials}

Hydroxyethyl cellulose and Sodium Carboxymethyl cellulose were taken in 3:2 ratios and dissolved in deionized water at $60^{\circ} \mathrm{C}$ for 8 hours. $20 \% \mathrm{w} / \mathrm{w}$ of Citric acid and $80 \% \mathrm{w} / \mathrm{v}$ Glycerol anhydrous purchased from SRL chemicals were used as the cross-linker and as plasticizer respectively. The homogenous mixture was poured in petri plates and dried in oven at $60^{\circ} \mathrm{C}$ for 12 hours to get a flexible and transparent film as shown in Fig. 1.
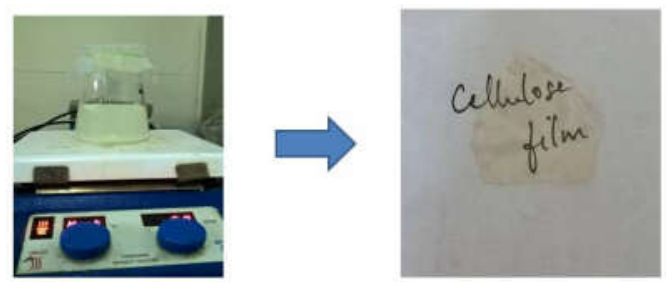

Figure 1: Synthesized transparent cellulose composite material

\section{Results and Discussion \\ Electrochemical Analyses}

Electrochemical studies of the test samples were carried out by electrochemical workstation (PGSTAT model $302 \mathrm{~N}$, Metrohm Autolab B. V, Netherlands) controlled by personal computer with NOVA 2.0 software. Three electrode electrochemical glass cells were used, comprising of Saturated Calomel Electrode (SCE) and a platinum foil as reference and counter electrodes respectively and the test sample as working electrode. Square wave analysis was performed with various additions of ethanol concentrations within a voltage range of $-0.5 \mathrm{~V}$ to $+1 \mathrm{~V}$. Chrono-amperometric analysis was performed at positive and negative voltages ( $0.5 \mathrm{~V}$ and $-0.1 \mathrm{~V}$ respectively) with gradual addition of ethanol at constant ( 30 seconds) time intervals. EIS measurement was performed in the frequency range $0-100 \mathrm{KHz}$ with sinusoidal potential of $0.5 \mathrm{~V}$ applied. The sample was immersed in artificial sweat electrolyte ( $\mathrm{pH}$ 8) for 15 minutes before the start of the experiment to get a stable OCP. The Nyquist plot for various addition of ethanol and the corresponding change in charge transfer resistance was determined. Cyclic voltammetry was measured within the range of $-2 \mathrm{~V}$ to +0.5 $\mathrm{V}$ with a scan rate of $0.01 \mathrm{~V} / \mathrm{s}$.

\section{Preparation of artificial sweat}

Artificial sweat was prepared as follows [22] (Table 1)

Table 1: Chemicals used for the preparation of artificial sweat

\begin{tabular}{cc}
\hline Chemicals & Quantity (g/L) \\
\hline $\mathrm{NaCl}$ & 7.5 \\
$\mathrm{KCl}$ & 1 \\
Urea & 1 \\
Lactic acid & 1 \\
$\mathrm{NH}_{3}$ added to adjust $\mathrm{pH}$ to 8 \\
\hline
\end{tabular}

\section{Swelling and Degradation}

The synthesized cellulose composite material's stability in water was determined by studying the swelling and degradation pattern of the material in artificial sweat. It was found that the material completely loses it stability from Day 12 onwards as seen in the Fig. 2. The material becomes like a gum in water due to excessive absorption of water. Due to hydrolysis $-\mathrm{OH}$ groups in the glycosidic backbone of the cellulose material are broken thus leading to the degradation of the material. Due to cross linking in the composite material this process occurs slowly.

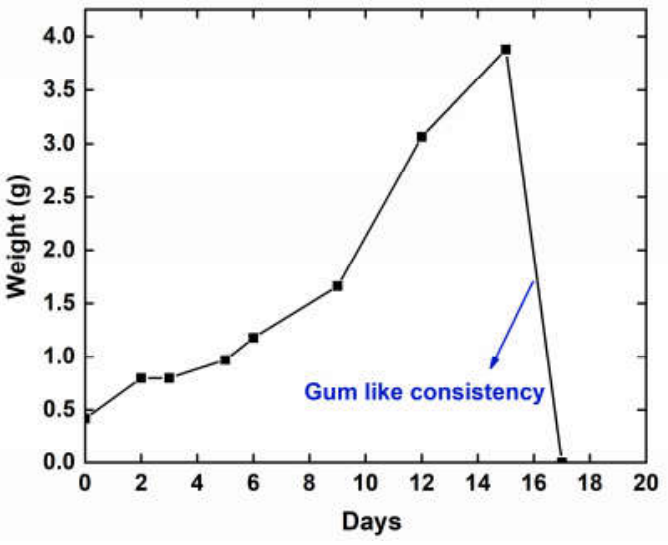

Figure 2: Swelling and degradation of the cellulose composite material

\section{Square-wave voltammetry}

Square wave analysis was performed for the material under the potential window $-0.5 \mathrm{~V}$ to $1 \mathrm{~V}$ (Fig. 3). With periodic addition of known concentration of ethanol in artificial sweat as the electrolyte solution, the current measurement was performed; this was also performed for bare cellulose film as well. For every addition of ethanol 


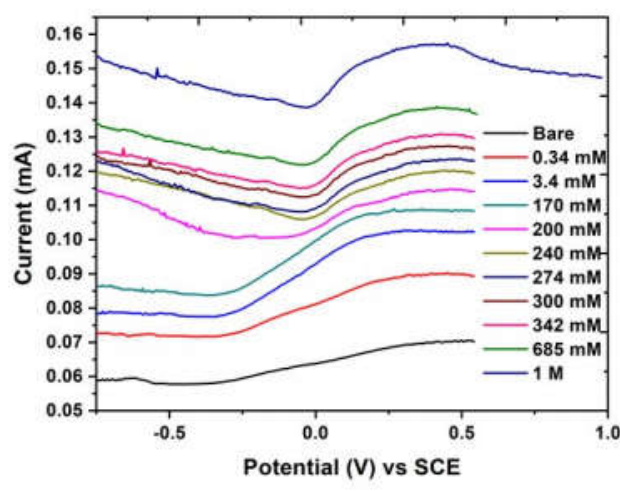

(a)

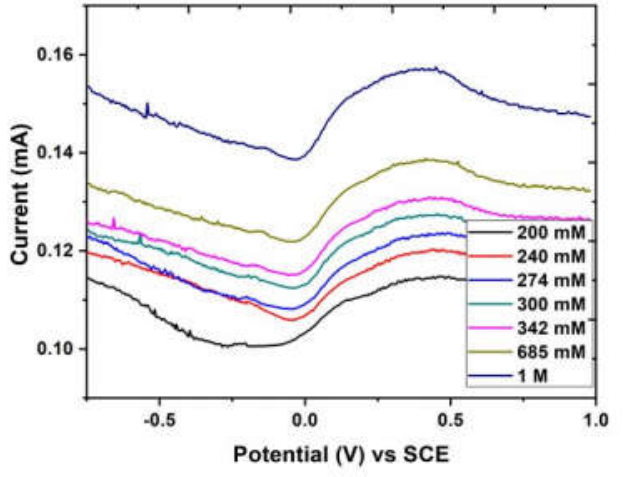

(b)

Figure 3: Square wave analysis for the cellulose composite material (a) showing full range of concentrations of ethanol (b) showing the significant changes observed for particular concentration of ethanol

(0.34 mM- $1 \mathrm{M})$, we could observe distinct redox peaks in the range of $-0.5 \mathrm{~V}$ to $+1 \mathrm{~V}$ and oxidation peaks in the range of $-0.5 \mathrm{~V}$ to $+0.1 \mathrm{~V}$. The incoming ethanol gets reduced by the material (working electrode) into $\mathrm{H}_{3} \mathrm{O}^{+}$and $\mathrm{H}^{+}$ions in the buffer as per the reaction mechanism explained in Fig. 10. Significant redox peaks were observed for concentration of ethanol $200 \mathrm{mM}-1 \mathrm{M}$ (Fig. 3b).

\section{Chronoamperometric study}

Chronoamperometric study was performed for the cellulose composite material by addition of ethanol at various intervals. A gradual increase in current response with a constant applied voltage of $-0.1 \mathrm{~V}$ and $+0.5 \mathrm{~V}$ as seen in Fig. 4 \& 5 was observed.

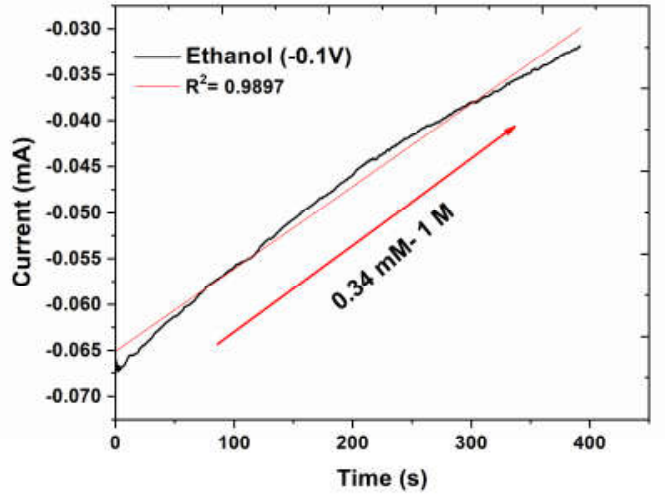

Figure 4: Chronoamperometric analysis of the cellulose composite material at $-0.1 \mathrm{~V}$ with varying concentration of ethanol

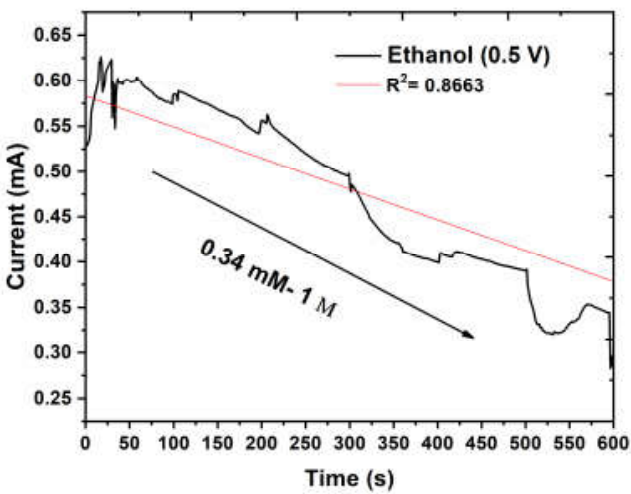

Figure 5: Chronoamperometric study of cellulose composite material at $0.5 \mathrm{~V}$
A stepwise increase in current response with addition of ethanol $(0.34 \mathrm{mM}-1 \mathrm{M})$ at every 30 seconds is evident from Fig. 4 \& 5. Minimum detection limit of the material was found at $0.34 \mathrm{mM}$ of ethanol and the range extends up to 1 M. Electron transfer observed between the analyte (artificial sweat) and the electrode was found to be steady which was observed at regular intervals of time. The cellulose composite material (working electrode) exhibited anodic nature at applied negative potential (Fig. 4) where oxidation (removal of electrons) takes place. Whereas, when positive potential is applied to the material, reverse reaction can be observed (Fig. 5) wherein the material transforms into a cathode and reduction (gain of electrons) occurs. Linear fit for the negative potential and positive potential was plotted and the $\mathrm{R}^{2}=0.9897$ and $\mathrm{R}^{2}=0.8663$ respectively.

The reaction observed here with ethanol is explained as follows:

a) Ethanol is an ionic solution which carriers a charge that reacted with the glycerol molecules to transfer $\mathrm{H}^{+}$ions.

b) Ethanol being an ionic liquid by itself and having selfionization tendency has given rise to this change in the material's ionic stand with applied negative and positive voltage.

\section{Electrical impedance spectroscopy analysis}

Electrochemical Impedance spectroscopy (EIS) is used for measuring the impedance of a material at a large frequency range of applied AC potential [23]. It is also known to be an effective technique to measure the interfacial electrontransfer resistance between an electrode and an analyte [24]. The Nyquist plot of EIS (Fig. 6) measurement provides an important information about the Faradaic chargetransfer occurring between the surface of the working electrode and the analyte in an ambient buffer solution. In Fig. 6, we could observe a clear decrease in impedance on addition of $3.4 \mathrm{mM}$ ethanol to the buffer solution (artificial sweat). The impedance is seen to be steadily decreasing when the concentration of ethanol in the artificial sweat was gradually increased. The $\mathrm{R}^{2}$ value for the linear fit of the various addition of ethanol was calculated as 0.9235 (Fig. 7).

EIS measurement was analyzed for the cellulose composite material at a frequency range of $0-100 \mathrm{KHz}$. For the first run with 0 concentration of ethanol, impendence was observed at $300 \mathrm{Ohm}$ and it drastically decreases to 80 
Ohm upon addition of $3.4 \mathrm{mM}$ ethanol. This shows that the electron transfers between the material's surface and the analyte is happening in higher rate. The release of $\mathrm{H}^{+}$ molecules from the material's surface with the interaction of ethanol gives rise to an increase in electron conductivity in the analyte which is then detected by the electrode. The freely moving charge carriers present in the buffer solution are the reason behind the steady increase in the electron conductivity of the electrolyte [25]. In Fig. 6, two semicircles are visible, one at higher frequency range and the other one at lower frequency range, the resistance at higher frequency range is higher than that of the resistance at the lower frequency range as observed in Fig. 6, this phenomenon indicates that the material is polycrystalline in nature [26].

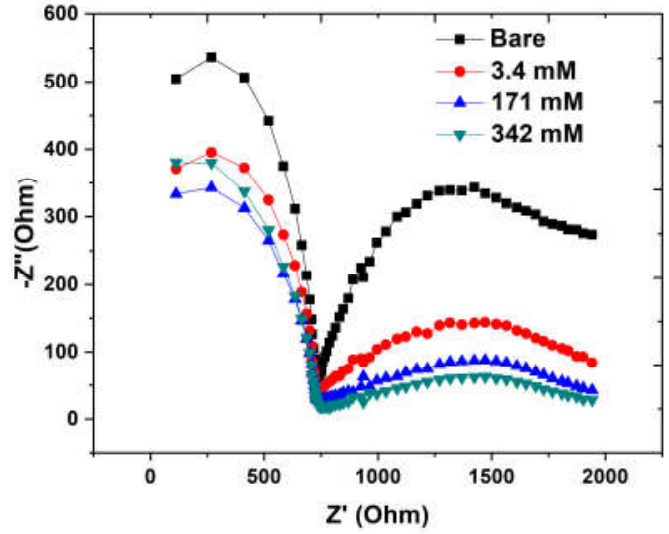

Figure 6: Impedance measurement taken at various concentration of ethanol

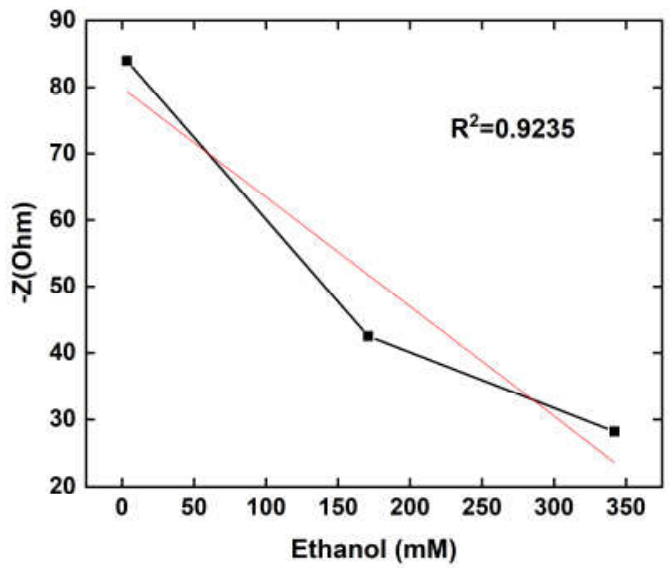

Figure 7: Linear fit for the impedance at various concentration of ethanol

\section{Cyclic Voltammetry analysis}

Cyclic voltammetry was performed for the material in artificial sweat at pH 8 (Fig. 8). The scan rate was set at $100 \mathrm{mV} / \mathrm{s}$ and the potential window was kept in the range of $-2 \mathrm{~V}$ to $0.5 \mathrm{~V}$. It was scanned at various concentrations of ethanol. Significant peaks were observed at $3.4 \mathrm{mM}$ and $342 \mathrm{mM}$ concentration of ethanol. The oxidation peak was observed at approximately $0.2 \mathrm{~V}$, whereas; the reduction peak could be seen at $-2 \mathrm{~V}$. In this context, oxidation implies that there is removal of electrons from the electrolyte whereas; reduction is the gain of electrons by the electrolytic solution. This is a continuous reaction taking place between the working electrode (cellulose composite material) and the electrolyte. The linear fit was calculated for the CV measurements with respect to the concentration of ethanol and the corresponding $\mathrm{R}^{2}$ value was found to be 0.9642 (Fig. 9).

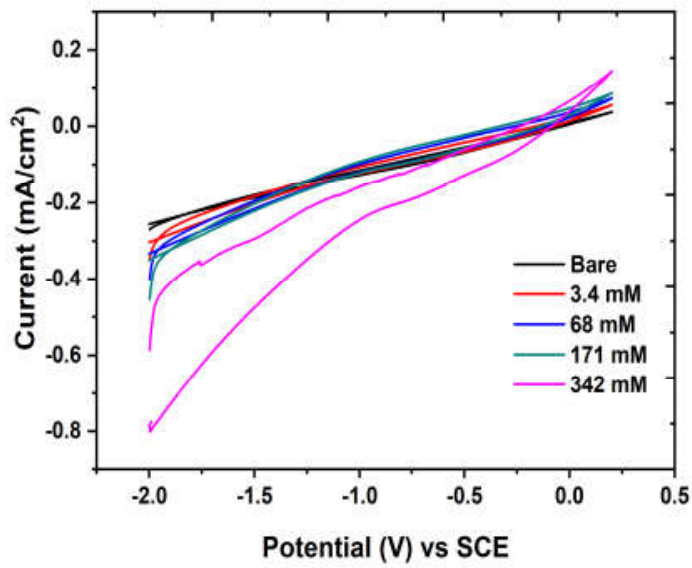

Figure 8: Cyclic-voltammetry measurement for various concentration of ethanol

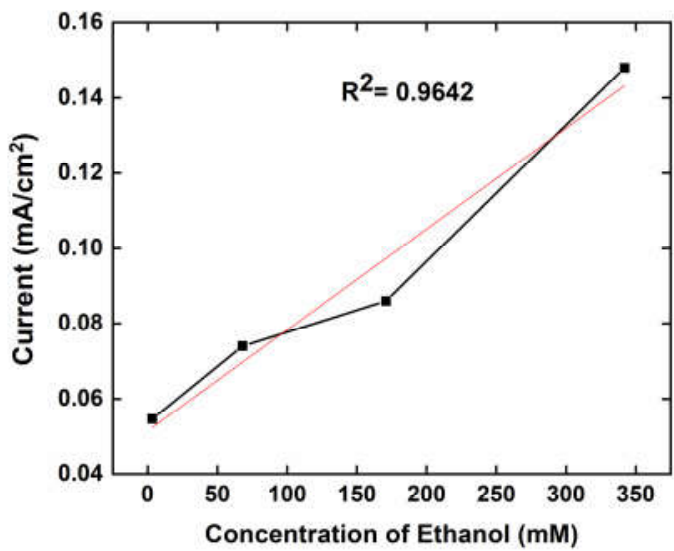

Figure 9: Linear Fit of the cyclic-voltammetry measurement for various concentration of ethanol

\section{Reaction mechanism}

The observed current response upon the addition of ethanol could be due to the presence of unreacted glycerol molecules adhered on the surface of the cellulose composite material which interacts with ethanol molecules to produce $\mathrm{H}_{2} \mathrm{O}$ (Fig. 10). However, we can observe that the water molecules are always found in dissociated state in aqueous solutions. The auto-ionization usually takes place in the form of hydronium $\left(\mathrm{H}_{3} \mathrm{O}^{+}\right)$and hydrogen $\left(\mathrm{H}^{+}\right)$ions [25]. The $\mathrm{H}_{2} \mathrm{O}$ molecules released from the cellulose composite material further deprotonates into $\mathrm{OH}^{-}$and $\mathrm{H}^{+}$ ions wherein the hydrogen ions again protonate with other water molecules to release $\mathrm{H}_{3} \mathrm{O}^{+}$(hydronium) and $\mathrm{H}^{+}$ (hydrogen ion).

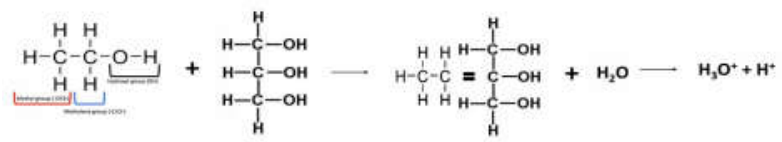

Figure 10: chemical reaction between ethanol and the surface glycerol molecules

\section{Fourier Transform Infra-red spectroscopy}

FTIR analysis was performed for the cellulose composite material before and after soaking with ethanol as shown in the Fig. 11. It is found to be in good agreement with the reaction mechanism proposed in Fig. 10. The interaction 
between the glycerol and ethanol molecules has significantly increased the $\mathrm{C}=\mathrm{C}$ functional groups $\left(1650 \mathrm{~cm}^{-}\right.$ 1) and the hydroxyl groups $\left(\mathrm{OH}^{-}\right)\left(3290 \mathrm{~cm}^{-1}\right)$ in the material as evidenced from Fig. 11. Figure 11 shows the FTIR analysis of three different types of cellulose composite materials (viz., bare cellulose film, cellulose film soaked in ethanol in dry and wet form). The spectrum that shows the wet ethanol-soaked $(200 \mathrm{mM})$ cellulose composite material has detected only the ethanol in the material and it can be noticed that the mono-substituted alkene $(\mathrm{C}-\mathrm{H})$ group $\left(1396 \mathrm{~cm}^{-1}\right)$ is seen only with the wet sample that apparently goes missing when the ethanol gets dried out of the cellulose composite material which implies that it either reacted with the other methyl groups in the material or just evaporated. The sudden appearance of a mono-substituted alkene group could be due to the immediate ejection of $\mathrm{H}_{2} \mathrm{O}$ molecules when ethanol comes in contact with the material leaving behind monosubstituted alkenes. In the second (dry-ethanol soaked) cellulose spectrum we can see intensified peaks at 1035 $\mathrm{cm}^{-1}, 1650 \mathrm{~cm}^{-1}$ and $3290 \mathrm{~cm}^{-1}$. These peaks have found to have higher intensity when compared to the pristine cellulose composite's FTIR spectrum indicating that there was an interaction with the additional alcohol molecules with the material's surface as shown in Fig. 11 by increasing the $\mathrm{C}-\mathrm{C}$ bonds and $\mathrm{C}-\mathrm{H}$ bonds and hydroxyl groups $(-\mathrm{OH})$ bonds. Whereas the intensity difference found for $\mathrm{C}-\mathrm{H}$ bonds are not so significant because of the quick evaporation of ethanol but the increase in intensity of $\mathrm{C}=\mathrm{C}$ bonds show that there has been chemical reaction between glycerol and alcohol.

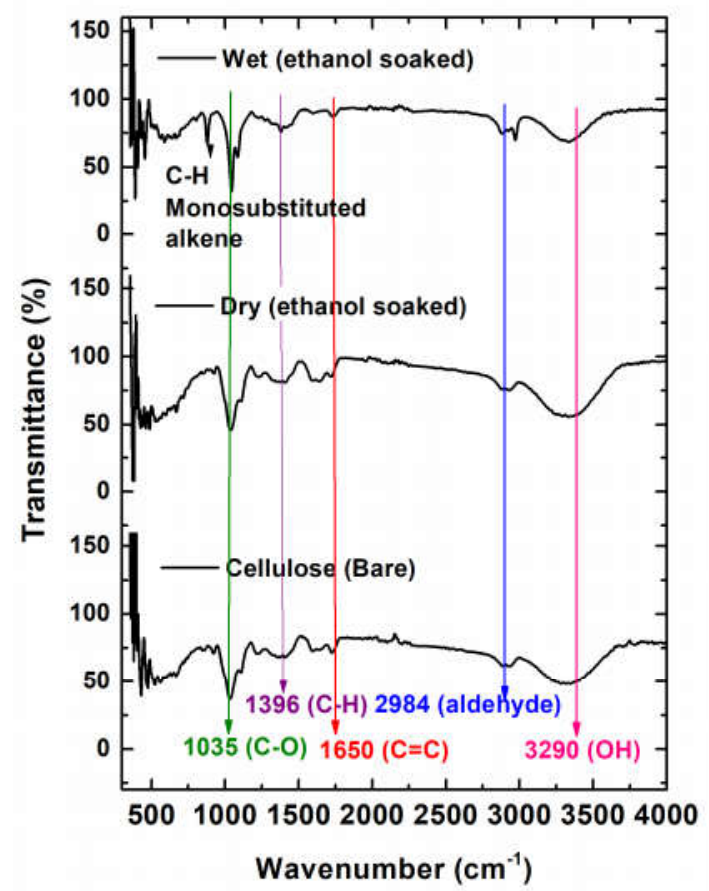

Figure 11: FTIR analysis for the cellulose membrane taken for bare, ethanol soaked and dried, wet ethanol soaked

\section{Conductivity measurement}

The material was experimented with addition of ethanol in the absence of externally applied potential for the material (Fig. 12). Due to the presence of artificial sweat as the electrolyte which predominantly has $\mathrm{NaCl}$ in it, the electrical conductance of the buffer solution can be determined by a conductivity meter. The addition of ethanol has significantly increased the conductivity of the electrolyte because the ethanol by itself is an ionic solution, hence it is electrically conductive. The increase in conductivity that is observed in Fig. 12 could because due

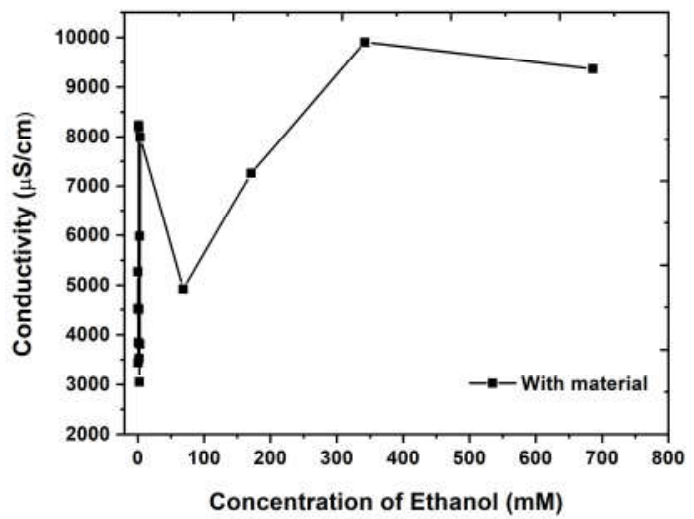

Figure 12: Conductivity measurement without applied voltage performed for varying ethanol concentration with the cellulose composite material

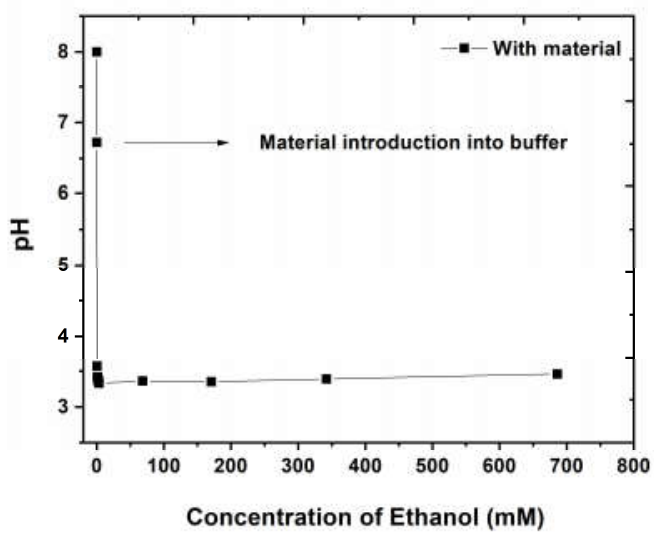

Figure 13: $\mathrm{pH}$ measurements for the cellulose composite material at varying ethanol concentration

to the self-ionization of the ethanol molecules in the electrolyte [26]. Here, we see steady increase in electrical conductance because of the self-ionization nature of both water molecules as well as ethanol molecules. Beyond a certain point the conductivity meter we have used is unable to detect the electron transfer and hence the value seems to be decreasing as it gets saturated. The electrical conductivity of the solution measured with the help of conductivity meter works on the principle of measuring the current output based on the ionic strength of the solution. Whereas in an electrochemical analyzer we have a 3-probe system that measures the electron transfer occurring between the working electron and the electrolyte with every addition of analyte that initiates a redox reaction (electron gain or electron loss) from the working electrode which is detected by the reference electrode that is converted into current output. This is called Faradaic principle. Hence this experiment was conducted to prove with examples to show the difference between a simple conductivity meter and an electrochemical system to measure electron transfer mechanism between a material and the analyte and comparing their efficiency.

\section{pH measurement}

The $\mathrm{pH}$ measurement (Fig. 13) on the other hand has shown to be in agreement with the reaction mechanism 
proposed in Fig. 10. The gradual drop in $\mathrm{pH}$ with the addition of the analyte shows that there is a significant rise in $\mathrm{H}^{+}$ions in the electrolyte which is possibly due to the following reasons:

a) Being an ionic solution ethanol dissociates in water into $\mathrm{H}^{+}$and $\mathrm{H}_{3} \mathrm{O}^{+}$ions thereby decreasing the $\mathrm{pH}$

b) Also due to the constant incoming of ethanol molecules there is a rapid electron transfer between water and ethanol molecules that increases the $\mathrm{H}^{+}$ ions in the electrolyte

c) And the release of water molecules from the material also contributes to the additional $\mathrm{H}^{+}$ions in the electrolyte that contributes to the drop in $\mathrm{pH}$.

All these together have increased the number of $\mathrm{H}^{+}$ions which has drastically brought down the $\mathrm{pH}$ of the solution. Since $\mathrm{pH}$ detects only the number of $\mathrm{H}^{+}$ions the above are the reasons that could possibly cause the decrease in $\mathrm{pH}$. This seconds the reactions mechanism that is proposed in Fig. 10. The reason behind stable $\mathrm{pH}$ maintained beyond pH 4.7 could be interpreted as follows:

a) The number of $\mathrm{H}^{+}$ions in the solution has been saturated and there are no electron transfers that occur between ethanol and water molecules beyond that point

b) Or because the $\mathrm{pH}$ meter used has a limitation to detect only up to a certain concentration of $\mathrm{H}^{+}$.

The working principle of the conductivity meter is by merely converting the ionic strength of the solution into current. The basic principle of conductivity meter is to measure the electrical conductance of the solution by applying a potential through 2 electrodes that is inserted into the solution. Also, the working methodology of an electrochemical system relies upon Faradaic principle wherein the electron transfer occurring between the working electrode and the electrolyte (oxidation and reduction) is detected with the help of a 3-electrode system that gives an increase of decrease in current output respectively.

\section{Conclusions}

The synthesized flexible and biodegradable cellulose composite material exhibits ethanol detecting property which was characterized by electrochemical analyses. The lowest detection range observed was $0.34 \mathrm{mM}$ observed with chrono-amperometric and square wave measurements. The material showed cathodic and anodic behavior at positive and negative applied voltage respectively. The reaction mechanism behind the electron transfer occurring with the working electrode is the dissociation of $\mathrm{H}_{2} \mathrm{O}$ molecules (ejected by the reaction between glycerol and ethanol) in the solution into $\mathrm{H}^{+}$and $\mathrm{H}_{3} \mathrm{O}^{+}$. This is counter verified with the help of off-voltage measurements carried out with the help of conductivity meter at room temperatures which also indicated a significant drop in $\mathrm{pH}$ of the electrolyte with addition of alcohol in the presence of the cellulose composite material.

\section{Acknowledgement}

We thank DST-WOS(A) scheme for supporting our work with financial aid

\section{Conflicts of Interest:}

In view of this submission the authors declare that there are no conflicts of interest.

\section{References}

1. Liao $\mathrm{C}$, Mak $\mathrm{C}$, Zhang $\mathrm{M}$, et al., Flexible organic electrochemical transistors for highly selective enzyme biosensors and used for saliva testing. Adv Mater, 2015, 27(4):676-681.

2. Pavinatto FJ, Paschoal CWA, Arias AC, Printed and flexible biosensor for antioxidants using interdigitated ink-jetted electrodes and gravure-deposited active layer. Biosens Bioelectron, 2015, 67:553-559.

3. Wang Y, Ping J, Ye Z, et al., Impedimetric immunosensor based on gold nanoparticles modified graphene paper for label-free detection of Escherichia coli 0157: H7. Biosens Bioelectron, 2013, 49:492-498.

4. Dong S, Xi J, Wu Y, et al., High loading MnO2 nanowires on graphene paper: Facile electrochemical synthesis and use as flexible electrode for tracking hydrogen peroxide secretion in live cells. Anal Chim Acta, 2015, 853:200-206.

5. Panneer Selvam A, Muthukumar S, Kamakoti V, Prasad S., A wearable biochemical sensor for monitoring alcohol consumption lifestyle through Ethyl glucuronide (EtG) detection in human sweat. Sci Rep, 2016, 6:1-11.

6. Penza M, Cassano G, Aversa P, et al., Alcohol detection using carbon nanotubes acoustic and optical sensors. Appl Phys Lett, 2004, 85:2379-2381.

7. Chiam YS, Lim KS, Harun SW, et al., Conducting polymer coated optical microfiber sensor for alcohol detection. Sensors Actuators, A Phys, 2014, 205:58-62.

8. Vijayan S, Govind VT, Mathews M, Surendran S (2014) Alcohol Detection Using Smart Helmet System. Int J Emerg Technol Comput Sci Electron 8:190-195

9. Boffetta P, Hashibe M., Alcohol and cancer. Lancet Oncol, 2006, 7(2):149-156.

10. Goedde HW, Agarwal DP, Fritze G, et al., Distribution of ADH2 and ALDH2 genotypes in different populations. Hum Genet, 1992, 88(3):344-346.

11. Oze I, Matsuo K, Wakai $\mathrm{K}$, et al., Alcohol drinking and esophageal cancer risk: An evaluation based on a systematic review of epidemiologic evidence among the Japanese population. Jpn. J. Clin. Oncol, 2011, 41(5): 677-692.

12. Boyle P, Boffetta P, Lowen A, et al (2013) Alcohol: Science, policy and public health. NAD Nord Stud Alcohol Drugs . https://doi.org/10.2478/nsad-2013-0026

13. Singletary KW, Gapstur SM., Alcohol and breast cancer: Review of epidemiologic and experimental evidence and potential mechanisms. J. Am. Med. Assoc, 2001, 286(17): 2143-2151.

14. Hamid A, Wani NA, Kaur J., New perspectives on folate transport in relation to alcoholism-induced folate malabsorption - Association with epigenome stability and cancer development. FEBS J, 2009, 576(8):2175-2191.

15. Bagnardi V, Rota M, Botteri E, et al., Alcohol consumption and site-specific cancer risk: A comprehensive doseresponse meta-analysis. Br J Cancer, 2015, 112:580-593.

16. Fedirko V, Tramacere I, Bagnardi V, et al., Alcohol drinking and colorectal cancer risk: An overall and dose-Response meta-analysis of published studies. Ann. Oncol, 2011, 22(9):1958-1972.

17. Saladi RN, Nektalova T, Fox JL., Induction of skin carcinogenicity by alcohol and ultraviolet light. Clin. Exp. Dermatol, 2010, 35(1):7-11.

18. Hauke A, Simmers P, Ojha YR, et al., Complete validation of a continuous and blood-correlated sweat biosensing device with integrated sweat stimulation. Lab Chip, 2018, 18(24): 3750-3759.

19. Maity, D., Rajavel, K., \& Kumar, R. T. R., Polyvinyl alcohol wrapped multiwall carbon nanotube (MWCNTs) network on fabrics for wearable room temperature ethanol sensor. Sensors and Actuators B: Chemical, 2018, 261, 297306. 
20. Kekec, N. C., Kanik, F. E., Udum, Y. A., Hizliates, C. G., Ergun, Y., \& Toppare, L., A novel conducting polymer based platform for ethanol sensing. Sensors and Actuators B: Chemical, 2014, 193, 306-314.

21. Ramadoss P, Rahman MI, Perumal A, et al., Non-Invasive, Non-Enzymatic, Biodegradable and Flexible Sweat Glucose Sensor and Its Electrochemical Studies. ChemistrySelect, 2020, 5:11305-11321.

22. Mathew MT, Ariza E, Rocha LA, et al., TiCxOy thin films for decorative applications: Tribocorrosion mechanisms and synergism. Tribol Int, 2008, 41:603-615.

23. Hammond JL, Formisano N, Estrela P, et al., Electrochemical biosensors and nanobiosensors. Essays Biochem, 2016, 60(1): 69-80.

24. Fu J, Pang Z, Yang J, et al., Fabrication of polyaniline/carboxymethyl cellulose/cellulose nanofibrous mats and their biosensing application. Appl Surf Sci, 2015, 349:35-42.

25. Geissler PL, Dellago C, Chandler D, et al., Autoionization in liquid water. Science, 2011, 291(5511): 2121-2124.

26. Joshi M., Importance of Impedance Spectroscopy Technique in Materials Characterization: A Brief Review. Mech Mater Sci Eng. 2017, 10 :9. 